\title{
Detection of Dirofilaria immitis (Nematoda: Filarioidea) by Polymerase Chain Reaction in Aedes albopictus, Anopheles punctipennis, and Anopheles crucians (Diptera: Culicidae) From Georgia, USA
}

\author{
BETH LICITRA, ${ }^{1}$ ERIC W. CHAMBERS, ${ }^{1}$ ROSMARIE KELLY, ${ }^{2}$ AND THOMAS R. BURKOT ${ }^{1,3}$
}

J. Med. Entomol. 47(4): 634-638 (2010); DOI: 10.1603/ME09277

\begin{abstract}
Potential mosquito vectors of Dirofilaria immitis (Leidy) (Nematoda: Filarioidea), the causative agent of dog heartworm in the southeastern region of the United States, were collected with CDC light traps and gravid traps in seven counties in the state of Georgia, USA. The presence of $D$. immitis in these mosquitoes was detected by polymerase chain reaction using species-specific primers for the D. immitis surface or cuticular antigen. Overall, 1,574 mosquitoes of 13 species in seven genera were collected; $92 \%$ of the specimens were Aedes albopictus (Skuse), Aedes vexans (Meigen), or Anopheles punctipennis (Say). Ae. albopictus, An. punctipennis, and Anopheles crucians Wiedemann were positive for D. immitis DNA. Ae. albopictus had the highest maximum likelihood rate of infection (2.30\%; $95 \%$ confidence interval $[\mathrm{CI}]=1.15-4.00 \%)$ followed by An. crucians $(1.38 \%: 95 \% \mathrm{CI}=$ 0.04-6.93\%), and An. punctipennis (0.85\%: 95\% CI 0.03-4.29\%). The detection of D. immitis DNA in the heads and thoraxes of Ae. albopictus $(0.40 \% ; 95 \% \mathrm{CI}=0.12-2.02 \%)$ indicates that these mosquitoes can support the development of $D$. immitis to the infective stage 3 larvae.
\end{abstract}

KEY WORDS Dirofilaria immitis, Aedes albopictus, Georgia

Dog heartworm is caused by infection with Dirofilaria immitis (Leidy) (Nematoda: Filarioidea), a mosquitotransmitted parasite of canines found throughout the world (Acha and Szyfres 2003). Sixty-three species of mosquitoes are thought to be potential vectors of $D$. immitis, with 28 of these being found in the United States (Ludlam et al. 1970); 12 of these species [Aedes albopictus (Skuse), Aedes sollicitans (Walker), Aedes sticticus (Meigen), Aedes taeniorhynus (Christophers), Aedes trivitattus (Coquillett), Aedes vexans (Meigen), Anopheles bradleyi King, Anopheles crucians Wiedemann, Anopheles punctipennis (Say), Culex nigripalpis Theobald, Culex quinquefasciatus Say, and Culex salinarius (Coquillett)] are found in the southeastern United States and have been implicated as presumptive vectors of $D$. immitis (Lok et al. 2000 ). Although it was once believed that transmission occurred year-round in the warmer southern United States, recent studies have shown that Dirofilaria transmission may be seasonal in some warm climate states (Watts et al. 2001).

The D. immitis infections in canines have been historically common in the southeastern United

The findings and conclusions in this report are those of the authors and do not necessarily represent the views of the Centers for Disease Control and Prevention.

${ }^{1}$ Division of Parasitic Diseases, Centers for Disease Control and Prevention, 4770 Buford Hgwy., Mailstop F-42, Chamblee, GA 30341.

${ }^{2}$ Georgia Division of Public Health, Atlanta, GA 30303-3109.

3 Corresponding author, e-mail: tburkot@cdc.gov.
States. In 1968 in Atlanta, D. immitis infection rates of 5.4 and $12.5 \%$ were found in privately owned dogs and dogs in shelters, respectively (Thrasher et al. 1968). In North Carolina and Virginia, a $19 \%$ D. immitis infection rate in dogs was found (Falls and Platt 1982). The D. immitis infections in dogs residing in animal shelters in northwestern South Carolina ranged from $8.7 \%$ (1991-1992) to $12.7 \%(1999-2000)$ (Yabsley et al. 2004).

In canines, dog heartworm is a chronic infection by adult $D$. immitis worms within the right ventricle and pulmonary arteries. In humans, infection with $D$. immitis can result in pulmonary dirofilariasis, a benign condition in which the parasites migrate to the heart and die and are then carried to the lungs. In the lung the parasites aggregate to form a pulmonary nodule (1-4 cm) known as a "coin" lesion (Acha and Szyfres 2003). These coin lesions can be mistaken for pulmonary malignancies and are often removed unnecessarily (Rodrigues-Silva et al. 1995). Although infections in humans are rare, four cases of human dirofilariasis were reported from Savannah, GA, between 1992 and 1999 (Echeverri et al. 1999).

Traditionally, field studies to incriminate vectors or to determine seasonality and intensity of transmission involved the dissection of large numbers of field-collected mosquitoes to determine whether a mosquito species was naturally infected with the infectious stage 3 larvae (L3) of D. immitis, which is transmitted from the vector to a vertebrate during blood feeding. 


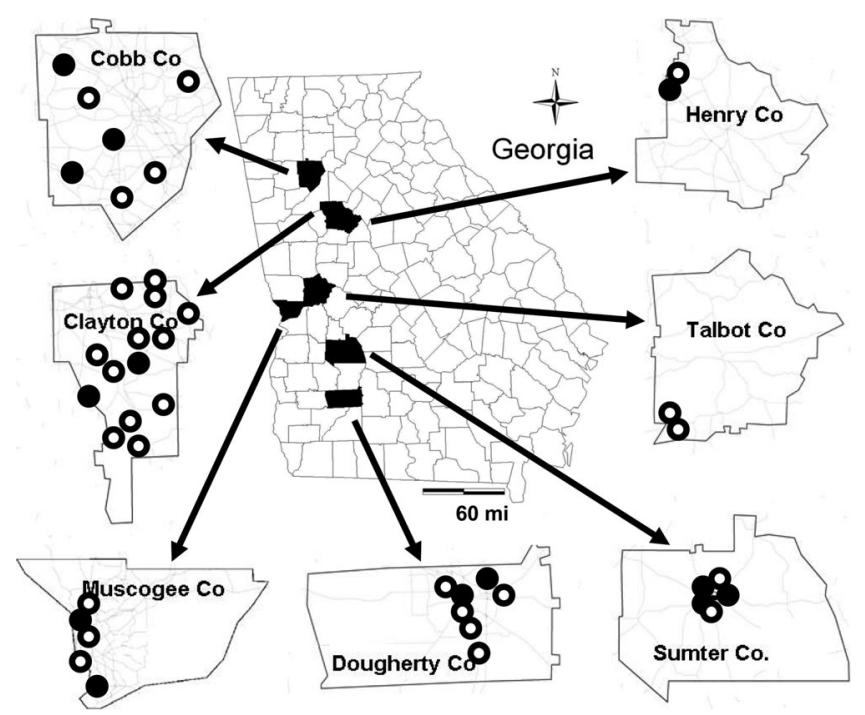

Fig. 1. Map of the state of Georgia with locations of the seven counties where mosquitoes were analyzed for D. immitis DNA. Approximate locations where all mosquitoes were negative for D. immitis DNA are shown as empty circles. Approximate locations with at least one mosquito positive for D. immitis DNA are shown as solid circles (map created 2009; source Georgia Division of Public Health: Centers for Disease Control and Prevention; Transverse Mercatur projection; UTM 1983, zone 16). Distance scale is for the map of Georgia.

It is logistically challenging to perform an adequate number of dissections for these studies and technically difficult to identify the L3s of different Dirofilaria species by morphology (Sauerman and Nayar 1983). However, sensitive and specific polymerase chain reaction (PCR)-based assays to circumvent these obstacles have been developed to identify $D$. immitis DNA in pools of mosquitoes (Scoles and Kambhampati 1995, Watts et al. 2001).

The current study identified potential mosquito vectors of $D$. immitis in Georgia, USA, by estimating the parasite infection rate by PCR analysis using specific primers to detect $D$. immitis DNA and emphasized the identification of D. immitis in non-Culex species (which constitute 10 of the 12 presumptive vectors of $D$. immitis in the southeastern United States; Lok et al. 2000) that were available from collections performed as part of a West Nile virus (family Flaviviridae, genus Flavivirus, WNV) study. PCR analysis of only the mosquito head and thorax was also conducted to identify mosquitoes that may be potentially infective (because only D. immitis DNA from L3s should be present in a vector mosquito's head and thorax).

\section{Materials and Methods}

Mosquito Collections. Mosquitoes were collected using both CDC light traps and gravid traps at 49 sites in seven counties in Georgia as part of the state WNV surveillance program (Fig. 1). Mosquitoes were collected between 29 September 2006 and 13 October 2006 and again between 11 May 2007 and 25 July 2007. Culex spp. [other than Culex erraticus (Dyar \& Knab)] were not available for screening for $D$. immitis because they had been processed previously by the Georgia
Division of Public Health for WNV detection and then discarded. Mosquitoes were pooled by species, trap location, and date of collection and were stored at $-80^{\circ} \mathrm{C}$ until analyzed for $D$. immitis.

Extraction of DNA From Mosquitoes. DNA from mosquito pools was extracted using the DNeasy kit (QIAGEN, Valencia, CA) protocol, with the only modification being that the column was washed twice with buffer AW1 and once with buffer AW2 before DNA was eluted from the column with $125 \mu \mathrm{l}$ of AE elution buffer. The purified DNA then was used for the PCR assay.

Extraction Controls. A negative DNA extraction control from a pool of noninfected laboratory-reared Ae. albopictus mosquitoes was run with each set of PCR reactions. In addition, a positive DNA extraction control and positive PCR control were included with each set of PCR reactions. The positive DNA extraction control consisted of a pool of laboratory-reared Ae. albopictus mosquitoes spiked with three to six $D$. immitis L3s (larvae provided by the National Institutes of Health/National Institute of Allergy and Infectious Diseases Filariasis Research Reagent Repository Center). The positive PCR control consisted of three to six D. immitis L3s.

PCR Amplification With D. immitis Primers. PCR assays were modified from the reaction conditions first published by Scoles and Kambhampati (1995) as described by Chambers et al. (2009) using primers based on the tandemly repeated $D$. immitis surface or cuticular antigen present at 20-50 copies per haploid genome. The repeat sequence (GenBank No. M82811) of this surface protein is 133 amino acids long with a molecular weight of $15 \mathrm{kDa}$ (Culpepper et al. 1992, Poole et al. 1992). Each 50- $\mu$ l PCR reaction 
Table 1. Filarial D. immitis DNA-positive rates in mosquitoes collected in Georgia, USA

\begin{tabular}{|c|c|c|c|c|c|}
\hline Species & Tested (no.) & Pools (no.) & $\begin{array}{l}\text { Avg. no. } \\
\text { mosquitoes per pool }\end{array}$ & $\begin{array}{c}\text { D. immitis } \\
\text { DNA-positive rate }\end{array}$ & $95 \% \mathrm{CI}$ \\
\hline Ae. albopictus & 604 & 98 & 6.2 & 2.30 & $1.15-4.00$ \\
\hline Ae. vexans & 551 & 54 & 10.2 & 0 & \\
\hline An. punctipennis & 121 & 20 & 6.1 & 0.85 & $0.03-4.29$ \\
\hline An. crucians & 77 & 10 & 7.7 & 1.38 & $0.04-6.93$ \\
\hline Anopheles quadrimaculatus Say & 23 & 7 & 3.3 & 0 & \\
\hline Cx. erraticus & 10 & 5 & 2 & 0 & \\
\hline Aedes canadensis (Theobald) & 4 & 2 & 2 & 0 & \\
\hline Psorophora columbiae (Dyar \& Knab) & 3 & 3 & 1 & 0 & \\
\hline Orthopodomyia signifera (Coquillett) & 3 & 3 & 1 & 0 & \\
\hline Psorophora ciliate (F.) & 2 & 1 & 1 & 0 & \\
\hline Aedes atlanticus Dyar \& Knab & 1 & 1 & 1 & 0 & \\
\hline Coquilletidia perturbans (Walker) & 1 & 1 & 1 & 0 & \\
\hline Uranotaenia sapphirina (Osten Sacken) & 1 & 1 & 1 & 0 & \\
\hline Total & 1,401 & 206 & 6.8 & 1.11 & $0.58-1.87$ \\
\hline
\end{tabular}

${ }^{a}$ Parasite DNA rates are max likelihood estimates as calculated by PoolScreen2 software (Katholi et al. 1995).

contained $1 \times$ Taq buffer (QIAGEN); $3 \mathrm{mM} \mathrm{MgCl}_{2}$; $0.20 \mathrm{mM}$ each dATP, dCTP, dGTP, and dTTP; 10 pmol of $D$. immitis surface antigen forward and reverse primers (5'-ACG TAT CTG AGC TGG CTC AC-3' and 5'-ATG ATC ATT CCG CTT ACG CC-3', respectively; Scoles and Kambhampati 1995), 1.25 U of HotStarTaq DNA polymerase, and $1 \mu \mathrm{l}$ of genomic DNA. PCR reactions were run on an I-Cycler (Bio-Rad Laboratories, Hercules, CA). The PCR conditions consisted of a single step of $95^{\circ} \mathrm{C}$ for $15 \mathrm{~min}$ and $50^{\circ} \mathrm{C}$ for 5 min (needed to activate the HotStar TaqDNA polymerase; S. Williams, personal communication), followed by 35 cycles of $72^{\circ} \mathrm{C}$ for $1 \mathrm{~min}, 90^{\circ} \mathrm{C}$ for $1 \mathrm{~min}$, and $50^{\circ} \mathrm{C}$ for $1 \mathrm{~min}$. The final step was a 5 -min extension at $72^{\circ} \mathrm{C}$. PCR products were size fractionated on $2 \%$ agarose gels stained with GelRed (Biotium, Hayward, CA). Agarose gels were run at $70 \mathrm{~V}$ for $1 \mathrm{~h}$ and visualized under UV light. Samples positive for the $D$. immitis surface antigen repeat yielded a 378-bp fragment after amplification. Positive samples were verified by repeating the PCR reaction in duplicate. A pool was considered positive if at least one of the two confirmatory repeat samples were also positive. Analysis of potential infections with $D$. immitis L3s was conducted by PCR using DNA extracted from the head and thorax of all Ae. albopictus captured in light and gravid traps during July 2007.

Estimation of Mosquito Infection Rates. Mosquito infection rates by PCR were calculated as maximum likelihood estimates, with 95\% confidence intervals (CI) based upon the likelihood ratio method using PoolScreen2 software (Katholi et al. 1995).

\section{Results and Discussion}

In the current study, 13 species in seven genera of mosquitoes (total of 1,401 mosquitoes in 206 pools) were analyzed from 49 trap locations in seven counties in Georgia (Fig. 1). Ninety-two percent of mosquitoes analyzed in this study were Ae. albopictus $(n=796)$, Ae. vexans $(n=551)$, and An. punctipennis $(n=122)$ (Table 1).

Three mosquito species were positive for D. immitis DNA: Ae albopictus, An. punctipennis, and An. crucians (Tables 1 and 2). This is the first study that reports evidence for the natural infection of mosquitoes with D. immitis in the state of Georgia. Many previous studies in other states reported mosquito species infected with filariid nematodes that were presumed (because of the difficulty of identifying $D$. immitis by morphology) to be D. immitis (reviewed by Lok et al. 2000 ), including the three species confirmed with $D$. immitis DNA in our study. Infected Ae. albopictus mosquitoes were identified from six of the seven counties in which mosquitoes were collected with $31 \%$ of the 49 collection sites $(n=15)$, yielding mosquitoes positive for D. immitis DNA, confirming the widespread distribution of this parasite (Fig. 1). Infected Ae albopictus were not found in Talbot Co. $(n=7)$. The estimated prevalence of D. immitis infection in Ae. albopictus for any larval stage was $2.30 \%(95 \% \mathrm{CI}=1.15-4.00 \%)$ (Table 1$)$, ranging from $0.92 \%(95 \% \mathrm{CI}=0.11-3.2 \%)$ in Clayton Co. to a high of $17.1 \%$ (95\% CI $=3.44-44.9 \%)$ in Sumter Co. among the six counties where evidence of infection was found. Overall, the prevalence of D. immitis L3s based on

Table 2. Numbers of wild-caught mosquitoes tested and number of PCR-pools positive for $D$. immitis (in parentheses) by year and month of collection

\begin{tabular}{|c|c|c|c|c|c|c|}
\hline Species & 2006 , Sept. & 2006, Oct. & 2007, May & 2007, June & 2007, July & Total \\
\hline Ae. albopictus & $59(2)$ & $217(5)$ & $25(3)$ & $41(2)$ & $262(1)$ & $604(13)$ \\
\hline Ae. vexans & 0 & $296(0)$ & $196(0)$ & $59(0)$ & 0 & $551(0)$ \\
\hline An. punctipennis & 0 & 7 & $113(1)$ & $1(0)$ & 0 & $121(1)$ \\
\hline An. crucians & 0 & 0 & 77 (1) & 0 & 0 & 77 (1) \\
\hline Others & 0 & $2(0)$ & $30(0)$ & $15(0)$ & $1(0)$ & $48(0)$ \\
\hline Totals & $59(2)$ & $515(5)$ & $441(5)$ & $116(2)$ & $263(1)$ & $1401(15)$ \\
\hline
\end{tabular}


detecting $D$. immitis DNA in the heads and thoraces of Ae. albopictus was $0.40 \%(95 \% \mathrm{CI}=0.12-2.02 \%)$.

An. punctipennis was collected from Dougherty, Muscogee, Sumter, and Talbot counties. A single D. immitis-positive pool was detected in the town of Americus in Sumter Co. The overall maximum likelihood of infection in An. punctipennis was $0.85 \%$ (95\% $\mathrm{CI}=0.03-4.29 \%)($ Table 1$)$. A single infected pool of An. crucians was found from Sumter Co.; (the only county in which this mosquito was collected). The maximum likelihood of infection was $1.38 \%(95 \% \mathrm{CI}=$ $0.04-6.93 \%)$. D. immitis DNA was not detected in any of the other 10 mosquito species tested (Table 1), including Ae vexans $(n=551)$, which was reported as a presumed vector in studies in 10 states, including the neighboring state of Alabama (Lok et al. 2000).

Ae. albopictus has been implicated previously as both an experimental and natural vector of $D$. immitis in Louisiana, Florida, and Italy (Comiskey and Wesson 1995, Nayar and Knight 1999, Cancrini et al. 2003). Results from our study confirm that not only is $A e$. albopictus capable of becoming infected with D. immitis in Georgia but also Ae. albopictus can support the development of $D$. immitis to the L3, based on the presence of D. immitis DNA in the head and thorax of this mosquito. Infection rates were higher in Ae. albopictus than in either of the other two D. immitis DNA-positive species, An. crucians and An. punctipennis. Ae. albopictus positive for D. immitis DNA were found in six of the seven counties that were sampled, but An. crucians- and An. punctipennis-positive mosquitoes were only detected in Sumter Co., although An punctipennis was collected in four counties albeit in limited numbers $(n<5)$ except for Sumter Co. where 113 An. punctipennis were collected and which yielded the D. immitis DNA-positive pool). Although the data presented cannot confirm whether transmission of dog heartworm occurs throughout the year in Georgia, infected mosquitoes were detected in each month in which samples were collected as early as 11 May and as late as 13 October, the latest and earliest dates when mosquitoes were collected, indicating a minimal 6-mo transmission season (Table 2).

Ae. albopictus was first detected in the continental United States in 1985 in Harris Co., TX (Sprenger and Wuithiranyagool 1986), and by 1997 it had spread to 678 counties in 25 states, including Georgia (Moore and Mitchell 1997). Ae. albopictus readily feeds on a variety of mammals, including dogs and humans (Savage et al. 1993), and Ae. albopictus is now known to support the development of D. immitis to the L3. In addition to the daytime feeding Ae. albopictus, An. punctipennis, and An. crucians, two dusk- and nighttime-feeding mosquitoes, were identified as infected with $D$. immitis by DNA analysis. The sampling biases associated with the mosquito collection methods (e.g., light and gravid traps may under-sample some species, including Aedes triseriatus Say, a presumptive D. immitis vector in the southeastern United States; Lok et al. 2000), as well as the unavailability of Ae. triseriatus, Cx. nigripalpis, Cx. salinarius, and Cx. quinquefasciatus for analysis, and the small numbers of mosquitoes from nine other species analyzed ( $n<24$ for each of these nine species), suggests that our study probably underestimated the number of species of dog heartworm vectors in Georgia. However, finding D. immitis DNA in mosquitoes that seek bloodmeals during the day, at dusk, and at nighttime suggests that domestic pets in Georgia are potentially at high risk of being exposed to the bites of dog heartworm-infected mosquitoes at all times when outdoors. Further work is needed to confirm the vector status of An. crucians and An. punctipennis by detecting the presence of D. immitis L3s in these suspected vectors.

\section{Acknowledgments}

We thank the Filariasis Research Reagent Repository Center (University of Georgia, Athens, GA) for providing L3 stage D. immitis and Steve Williams (Smith College) for advice on PCR conditions.

\section{References Cited}

Acha, P. N., and B. Szyfres. 2003. Zoonoses and communicable diseases common to man and animals. Pan American Health Organization, Washington, DC.

Cancrini, G., A. Frangipane di Regalbono, I. Ricci, C. Tessarin, S. Gabrielli, and M. Pietrobelli. 2003. Aedes albopictus is a natural vector of Dirofilaria immitis in Italy. Vet. Parasitol. 118: 195-202.

Comiskey, N., and D. M. Wesson. 1995. Dirofilaria (Filarioidea, Onchocerciadae) infection in Aedes albopictus (Diptera, Culicidae) collected in Louisiana. J. Med. Entomol. 32: 734-737.

Culpepper, J., R. B. Grieve, L. Friedman, M. Mika-Grieve, G. R. Frank, and B. Dale. 1992. Molecular characterization of a Dirofilaria immitis cDNA encoding a highly immunoreactive antigen. Mol. Biochem. Parasitol 54: 5162.

Echeverri, A., R. F. Long, W. Check, and C. M. Burnett. 1999. Pulmonary dirofilariasis. Ann. Thorac. Surg. 67: 201-202.

Falls, R. K., and T. R. Platt. 1982. Survey for heartworm, Dirofilaria immitis, and Dipetalonema reconditum (Nematoda, Filarioidea) in dogs from Virginia and North Carolina. Am. J. Vet. Res. 43: 738-739.

Katholi, C. R., L. Toè, A. Merriweather, and T. R. Unnasch. 1995. Determining the prevalence of Onchocerca volvulus infection in vector populations by polymerase chain reaction screening of pools of black flies. J. Infect. Dis. 172: 1414-1417.

Lok, J. B., E. D. Walker, and G. A. Scoles. 2000. Filariasis, pp. 299-375. In B. F. Eldridge and J. D. Edman (eds.), Medical Entomology. Kluwer Academic Publishers, Dordrecht, The Netherlands.

Ludlam, K. W., L. A. Jachowski, Jr., and G. F. Otto. 1970. Potential vectors of Dirofilaria immitis. J. Am. Vet. Med. Assoc. 157: 1354-1359.

Moore, C. G., and C. J. Mitchell. 1997. Aedes albopictus in the United States: ten-year presence and public health implications. Emerg. Infect. Dis. 3: 329-334.

Nayar, J. K., and J. W. Knight. 1999. Aedes albopictus (Diptera: Culicidae): an experimental and natural host of Dirofilaria immitis (Filarioidea: Onchocercidae) in Florida, USA. J. Med. Entomol. 36: 441-448.

Poole, C. B., A. G. Gandea, 3rd, C. V. Maine, R. E. Jenkins, M. E. Selkrik, and L. A. McReynolds. 1992. Cloning of a 
cuticular antigen that contains multiple tandem repeats from the filarial parasite Dirofilaria immitis. Proc. Natl. Acad. Sci. USA 89: 5986-5990.

Rodrigues-Silva, R., H. Moura, G. Dreyer, and L. Rey. 1995. Human pulmonary dirofilariasis: a review. Rev. Inst. Med. Trop. Sao Paulo 37: 523-530.

Sauerman, D. M., and J. K. Nayar. 1983. a survey for natural potential vectors of Dirofilaria immitis in Vero-Beach, Florida. Mosq. News 43: 222-225.

Savage, H. M., M. L. Niebylski, G. C. Smith, C. J. Mitchell, and G. B. Craig, Jr. 1993. Host-feeding patterns of Aedes albopictus (Diptera: Culicidae) at a temperate North American site. J. Med. Entomol. 30: 27-34.

Scoles, G. A., and S. Kambhampati. 1995. Polymerase chain reaction-based method for the detection of canine heartworm (Filarioidea: Onchocercidae) in mosquitoes (Diptera: Culicidae) and vertebrate hosts. J. Med. Entomol. 32: 864-869.
Sprenger, D., and T. Wuithiranyagool. 1986. The discovery and distribution of Aedes albopictus in Harris County, Texas. J. Am. Mosq. Control Assoc. 2: 217-219.

Thrasher, J. P., K. G. Gould, M. J. Lynch, and C. C. Harris. 1968. Filarial infections of dogs in Atlanta, Georgia. J. Am. Vet. Med. Assoc. 153: 1059-1063.

Watts, K. J., G. R. Reddy, R. A. Holmes, J. B. Lok, D. H Knight, G. Smith, and C. H. Courtney. 2001. Seasonal prevalence of third-stage larvae of Dirofilaria immitis in mosquitoes from Florida and Louisiana. J. Parasitol. 87: 322-329.

Yabsley, M. J., C. Dresden-Osborne, E. A. Pirkle, J. M. Kirven, and G. P. Noblet. 2004. Filarial worm infections in shelter dogs and cats from northwestern South Carolina, USA. Comp. Parasitol. 71: 154-157.

Received 18 November 2009; accepted 25 February 2010 